\title{
Efecto de Seis Tipos de Sustratos y Tres Dosis de Ácido a Naftalenacético en la Propagación Vegetativa de Mortiño (Vaccinium floribundum Kunth)
}

\author{
Mgs. Vilma Fernanda Noboa Silva, \\ Escuela Superior Politécnica de Chimborazo, Ecuador
}

Doi: 10.19044/esj.2019.v15n12p359 URL:http://dx.doi.org/10.19044/esj.2019.v15n12p359

\section{Resumen}

El mortiño (Vaccinium floribundum Kunth) es un arbusto silvestre aún en proceso de domesticación en el país cuyo desarrollo es lento y requiere de estudios más profundos. Esta es una planta con valor social, cultural, ecológico, productivo y comercial, siendo una de las pocas alternativas para mejorar la sostenibilidad de los páramos en el Ecuador. El presente estudio estuvo dirigido a probar seis sustratos y tres dosis de ácido $\alpha$ naftalenacético con el fin de establecer un protocolo de propagación vegetativa. El diseño experimental aplicado es el de bloques completos al azar (BCA) con arreglo combinatorio $6 \times 3$ con 3 repeticiones. Las evaluaciones fueron realizadas a los 90, 120 y 150 días utilizando la prueba de Tukey al 5\%, donde se registró: porcentaje de prendimiento, número de brotes por estaca y longitud de brotes. En las evaluaciones realizadas el tratamiento T13 (50\% paja $+50 \%$ tierra de crecimiento natural de la especie, con dosis alta de ácido $\alpha$ naftalenacético A.N.A) fue mejor en cuanto a porcentaje de prendimiento y número de brotes por estaca ( $86.67 \%$ y 2.67 brotes/estaca) y en longitud de brotes el que mejor resultó con $1.2 \mathrm{~cm}$ fue el tratamiento T1 (100\% tierra de crecimiento natural con dosis alta de auxina), estos resultados confirman que la especie reacciona favorablemente en suelos de desarrollo natural y que en combinación con un medio de aireación (paja de páramo) el resultado es mejor. La dosis alta de auxina sintética A.N.A incidió para un mejor desarrollo de la planta, incluso en aquellos tratamientos donde la tierra natural de crecimiento de la especie no estuvo presente.

Palabras clave: Sustratos, ácido naftalenacético, propagación vegetativa, vaccinium floribundum, páramo 


\title{
Effect of Six Types of Substrates and Three Dose of a Naftalenacetic Acid in the Vegetative Propagation of Mortiño (Vaccinium floribundum Kunth)
}

\author{
Mgs. Vilma Fernanda Noboa Silva, \\ Escuela Superior Politécnica de Chimborazo, Ecuador
}

\begin{abstract}
The mortiño (Vaccinium floribundum Kunth) is a wild shrub still in the process of domestication in the country whose development is slow, and it requires more in-depth studies. This is a plant with social, cultural, ecological, productive and commercial value, being one of the few alternatives to improve the sustainability of the páramos in Ecuador. This paper focuses on testing six substrates and three doses of $\alpha$-naphthaleneacetic acid in order to establish a vegetative propagation protocol. The experimental design applied is randomized complete blocks (BCA) with combinatorial arrangement $6 \times 3$ with 3 repetitions. The evaluations were carried out at 90, 120, and 150 days using the Tukey test at 5\%. Here, the following parameter was recorded: percentage of capture, number of shoots per stake, and length of shoots. In the evaluations made, the T13 treatment $(50 \%$ straw $+50 \%$ natural growth soil of the species, with a high dose of $\alpha$-naphthaleneacetic acid ANA) was better in terms of percentage of shoot and number of shoots per stake (86.67\% and 2.67) (buds / stake). For the length of shoots, the best result with $1.2 \mathrm{~cm}$ was the $\mathrm{T} 1$ treatment $(100 \%$ natural growth soil with high dose of auxin). However, these results confirm that the species reacts favorably in soils of natural development. In combination with a means of aeration (páramo straw), the result is better. The high dose of synthetic auxin ANA has a significant effect on the development of the plant, even in cases where the natural soil of the growth of the species was not present.
\end{abstract}

Keywords: Substrates, naphthaleneacetic acid, vegetative propagation, vaccinium floribundum, paramo

\section{Introduction}

El páramo es un ecosistema de clima frío y es muy susceptible a los cambios en el uso de la tierra, por lo que su potencial para el uso productivo es limitado. Sin embargo, mucha gente de una gran riqueza cultural pero pobreza económica ha aumentado por necesidad el uso directo del páramo 
tanto para cultivos de sustento como para el pastoreo de sus animales domésticos,convirtiéndose así grandes extensiones de páramos en haciendas de ganadería extensiva o en plantaciones de pinos con dudoso éxito y claro impacto ambiental (Hofstede, 2002). Las reformas agrarias posteriores a 1960 conllevaron a transiciones significativas del uso de la tierra de páramo y el bosque nativo a pinares (Ross, et al., 2017).

Los páramos son y han sido el sustento de muchas personas desde hace mucho tiempo y su productividad se la puede plantear desde varios frentes, todos ellos interrelacionados: la productividad en términos puramente ecológicos, la productividad relacionada con la agrobiodiversidad nativa y exótica, y la productividad relacionada con los servicios ambientales potenciales o reales del ecosistema (Mena \& Hofstede, 2006). En los ecosistemas de páramo existe una diversidad de productos forestales no madereros con alto potencial para ser usados por la población humana. Para esto se hace necesario realizar estudios básicos, relacionados en aspectos de distribución geográfica, propagación, alternativas de uso y otros. Frente a este problema los productos forestales no madereros representan una alternativa valedera en el campo económico, ambiental y social (Aguirre, 1998).

Una especie promisoria con posibilidades de uso es el mortiño (Vaccinium floribundum Kunth), producto natural de los páramos ecuatorianos, cuyo potencial no ha sido aprovechado a pesar de que sus frutos son nutritivos y exclusivos de las montañas andinas, constituyendo una fuente de alimento para la población paramera. No se conoce que existan cultivos comerciales de esta especie, sino únicamente pequeñísimas parcelas y/o chaparros de montaña de páramo en los que la fruta crece en forma silvestre.

La importancia del mortiño en el páramo tiene dos aspectos generales:en primer lugar, es prácticamente la única especie que tiene un valor alimenticio directo para la gente. Por esta razón tiene consigo un significado social y (aunque muy poco) comercial. El consumo del mortiño en el Ecuador, aunque es básicamente en fresco en la elaboración de la tradicional colada morada, un plato típico ecuatoriano de la época del día de los difuntos a inicio del mes de noviembre (valor cultural), también se realiza en procesados como: mermeladas, vinos, harina, usando su cáscara como materia prima para la elaboración de medicamentos y el fruto en el teñido de textiles.

Un segundo aspecto que le da importancia al mortiño en el páramo, es el rol en el funcionamiento del ecosistema ya que, al tener su propio nicho ecológico, uno de los aspectos típicos del mortiño y de otros arbustos similares (casi únicamente ericáceas) es que tienen frutos comestibles para animales frugívoros; un aspecto no tan común en el páramo donde la mayoría de las plantas se dispersan de manera anemócora por viento y por esto no tienen frutas comestibles. Es por ello que gracias al mortiño y a la mayoría de 
ericáceas existen aves en el páramo y otros animales que viven de sus frutas (o, a su vez, de las aves que viven de las frutas).

En un estudio hecho por el convenio MAG/IICA llamado Identificación de mercados y tecnología para productos agrícolas tradicionales de exportación" en el 2001 se propone la promoción del mortiño del Ecuador como "Andean blueberry". Según Chad Finn en su artículo "Temperate Berry Crops" publicado en "blueberries.org" el mortiño es una fruta con interesante potencial en el mercado como una nueva fruta ("potencial new berry"), que podría cultivarse y promover su consumo en el mercado mundial debido a la amplia aceptación de especies muy similares.

Tomando en cuenta lo expuesto anteriormente, la finalidad de este trabajo es la de determinar el mejor tratamiento en cuanto a prendimiento y desarrollo de nuevas plántulas con el fin de ir estableciendo un protocolo de propagación del mortiño en el páramo del Ecuador y de, en el futuro, establecer cultivos comerciales de este fruto.

\section{Texto Principal}

\section{Descripción Botánica del Mortiño}

El mortiño pertenece al Reino Plantae, Filo Magnoliophyta, Clase Magnoliopsida, Orden Ericales, Familia Ericaceae, Nombre Científico Vaccinium floribundum, Sinonimia Vaccinium mortinia (Freire, 2004). Tiene diferentes nombres comunes en los países donde esta especie se encuentra, siendo llamada en Ecuador: mortiño, uva de los andes, manzanilla del cerro, raspadura quemada, uva del monte, en Colombia: agraz y en Perú: macha macha, congama, pushgay.

\section{Descripción Morfológica}

Arbustos ramificados, hojas muy pequeñas con el margen aserrado o crenado, nerviación pinnada, flores de menos de $1 \mathrm{~cm}$, solitarias o en racimos; tubo del cáliz articulado o no con el pedicelo, hipanto globoso, 5 lóbulos lanceolados; corola urceolada, blanca o rosada, con 5 lóbulos reflexos, estambres de $8-10$, del mismo largo que el tubo de la corola, filamentos libres, anteras con túbulos cortos, dehiscencia apical poricida; ovario ínfero, 5 locular, estilo ligeramente más largo que el tubo de la corola. El fruto es una baya (Ulloa \& Jorgensen, 1995).

\section{Composición y usos}

Las hojas contienen tanino, flavona, glucoquitina, arbutina e hidroquinina y los frutos por su lado contienen azúcar invertido, ácidos orgánicos, mirtilina, tanino, pectina y vitaminas B y C (CESA, 1993). De igual manera, el mortiño"es una buena fuente de fibra y vitamina C. Estudios 
recientes afirman que contiene igualmente propiedades que fortalecen la vista.

Un grupo de investigadores de la UN en Medellín, en cooperación con la Corporación Autónoma Regional del Centro de Antioquia (CORANTIOQUIA) y la Corporación Colombiana de Investigación Agropecuaria (CORPOICA), estudiaron el agraz o mortiño y en la conclusión del estudio publicada en marzo 2009 manifiestan que este es uno de los frutos con mayor potencial antioxidante de los hasta ahora estudiados por el grupo. De acuerdo con esto, comparativamente el mortiño tendría más presencia de polifenoles que otros frutos como la fresa, la curuba, la mora y la gran mayoría de frutas tropicales colombianas. La presencia de antioxidantes en los alimentos retarda y previene la oxidación de otras moléculas, y constituye un factor determinante para aprovechar en otros usos farmacológicos como el tratamiento para accidentes cerebro-vasculares $\mathrm{y}$ enfermedades neurodegenerativas.

Los investigadores consideran que a partir de este fruto se pueden encontrar grandes ventajas químicas y obtener, mediante procesos tecnológicos, productos fermentados de alto valor agregado como vinos, salsas y vinagres balsámicos. El fruto del mortiño se usa principalmente como alimento humano en jaleas, mermeladas, vino y harina (Cadme, 2001). Los frutos también se utilizan en la elaboración de postres, los mismos que se comercializan en prestigiosas pastelerías y heladerías, aunque el principal uso es en la tradicional colada morada en la época de difuntos a inicios de noviembre en el Ecuador. Los frutos también se utilizan para tinturajes de ropa de lana. En menor frecuencia se lo usa como leña.

\section{Materiales y Métodos}

El estudio se llevó a cabo en la Comunidad de Shobol Alto Guadalupe en la provincia de Chimborazo, con una altitud de $3.566 \mathrm{msnm}$, temperatura promedio de $11^{\circ} \mathrm{C}$, con $1000 \mathrm{~mm} /$ año de precipitación y humedad promedio del $75 \%$. Zona escogida por encontrarse en el páramo, ecosistema donde el mortiño se da de manera natural.

\section{Material experimental}

Se emplearon estacas de mortiño, fundas para propagación, regaderas, zaranda, pala, tijeras de podar, tijeras, carretilla, parafina, sarán, pala de desfonde, recipientes plásticos, fundas de basura, sacos de nylon, picadora de pasto, rótulos, libreta de apuntes, formato para evaluación y seguimiento del ensayo, lápiz, libro de campo, tablas de monte, listones de madera, clavos, martillo, serrucho, machete, regla, lupa, hojas de papel bond. 


\section{Equipos}

Los equipos utilizados fueron un computador, una cámara de fotos, un GPS y un vehículo.

\section{Insumos}

Tierra de páramo, paja de páramo, turba, ácido $\alpha$-Naftalenacético (ANA).

\section{Identificación de zonas para recolección}

Con el fin de identificar las localidades productoras del Vaccinium comestible se tuvieron acercamientos a dirigentes comunitarios, técnicos de la Reserva de Producción Faunística Chimborazo y el Parque Nacional Sangay, quienes nos facilitaron el acceso a las comunidades que son parte del área de influencia donde hay antecedentes de recolección de este fruto en la época de finados (inicios de noviembre).

Las visitas se planificaron para la época de floración durante los meses de agosto y septiembre.

Tabla 1. Lugares visitados donde se registra Vaccinium floribundum

\begin{tabular}{|c|c|c|c|}
\hline LUGAR & LATITUD & LONGITUD & ALTURA \\
\hline Puesto de control de la Reserva Chimborazo & $\mathrm{S} 01^{\circ} 30^{\prime} 16.92^{\prime \prime}$ & $\mathrm{W} 85^{\circ} 9^{\circ} 48.79^{\prime \prime}$ & $4384 \mathrm{msnm}$ \\
\hline Losan & 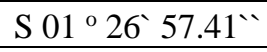 & $\mathrm{W} 85^{\circ} 12^{\prime} 40.9^{\prime \prime}$ & $4216 \mathrm{msnm}$ \\
\hline Key & 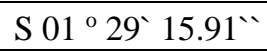 & $\mathrm{W} 78^{\circ} 56^{\prime} 22.58^{\prime \prime}$ & $3864 \mathrm{msnm}$ \\
\hline Puculpala & $\mathrm{S} 01^{\circ} 41^{\prime} 47.5^{\prime \prime}$ & $\mathrm{W} 78^{\circ} 34^{\prime} 40.7^{\prime \prime}$ & $3065 \mathrm{msnm}$ \\
\hline Ganquis & $\mathrm{S} 01^{\circ} 34^{\prime} 30.73^{\prime \prime}$ & $\mathrm{W} 78^{\circ} 51^{\prime} 57.14^{\prime \prime}$ & $3920 \mathrm{msnm}$ \\
\hline
\end{tabular}

Fuente: Visitas de campo

\section{Adecuación del sitio del ensayo}

Se hizo una cama de repique bajo nivel de $6 \mathrm{~m} \times 1 \mathrm{~m} \times 1 \mathrm{~m}$, donde con tablones de madera se elaboraron cajones de $0,8 \mathrm{~m}$ x $1 \mathrm{~m}$ para dividir los tratamientos con sus respectivas repeticiones. Con varillas y sarán se hizo un cubierta con la finalidad de proteger el ensayo del viento, heladas y granizo principalmente.

\section{Preparación de sustratos}

La tierra de páramo, turba y paja fueron extraídas de los páramos de San Juan, del sector donde crece el mortiño; posteriormente fueron cernidas con una zaranda. La paja fue cortada con tijeras y machete entre $1-3 \mathrm{~cm}$; luego se procedieron a realizar las tres mezclas necesarias para el ensayo $(50 \%$ Paja de páramo $+50 \%$ turba; $50 \%$ Paja de páramo $+50 \%$ tierra de páramo; $30 \%$ Paja de páramo $+35 \%$ tierra de páramo $+35 \%$ turba). 
Las fundas de propagación se llenaron de las mezclas descritas de manera tal que no quedaran espacios de aire en el interior, pero tampoco muy compactas ya que dificultaría la aireación.

La desinfección del sustrato se realizó con agua caliente a $80^{\circ} \mathrm{C}$ en una dosis de 3.33 litros $/ \mathrm{m}^{2}$ de fundas en la cama de repique con el fin de disminuir el ataque de organismos no benéficos que pudieran afectar la propagación.

\section{Obtención del material vegetativo}

Se recolectó el material vegetativo en la comunidad de Ganquis por tener mayor número de plantas productivas en relación a los otros lugares visitados. Para ello se cortaron ramas semileñosas de la parte media y apical del arbusto que presentaban signos de ser productivos con características fenotípicas deseables. Para poder evidenciarlo se cortaron las ramas en época de floración (agosto y septiembre).

Una forma de evitar la pérdida de humedad del material vegetativo hasta poder obtener las estacas y sembrarlas, es guardarlo en bolsas de plástico con papel periódico húmedo.

En el sitio del ensayo de propagación se cortaron con la tijera de podar estacas de las partes medias de las ramas que presentaron un diámetro de 0.4 $-1 \mathrm{~cm}$, con más de 2 yemas presentes y con una longitud de entre 4 a $6 \mathrm{~cm}$; el corte se lo hizo en forma biselada en la parte superior e inferior de las estacas.

\section{Dosis de ácido $\alpha$-naftalenacético}

En recipientes plásticos se prepararon las distintas dosis determinadas para el ensayo de Ácido $\alpha$-Naftalenacético (A.N.A) el cual es un fitorregulador hormonal, con actividad auxínica horizontal, que ejerce su acción en forma análoga a otros compuestos homólogos, como el ácido indol butírico (AIB) y/o el ácido indol acético (AIA), pero con mayor versatilidad y eficiencia que estos, ya que estimula el metabolismo de la planta en diversos eventos fisiológicos además del enraizamiento.

Tabla 2. Concentración de Ácido $\alpha$-Naftalenacético (A.N.A)

\begin{tabular}{|c|c|}
\hline DOSIS & CONCENTRACIÓN \\
\hline Recomendada & $0,05 \mathrm{~g} / \mathrm{cm}^{3}$ \\
\hline Alta & $0,1 \mathrm{~g} / \mathrm{cm}^{3}$ \\
\hline Media & $0,066 \mathrm{~g} / \mathrm{cm}^{3}$ \\
\hline Baja & $0,033 \mathrm{~g} / \mathrm{cm}^{3}$ \\
\hline
\end{tabular}

En estas soluciones se insertaron las partes inferiores de las estacas, sumergiendo la base vegetal por 16 horas para que estén listas para ser sembradas. 


\section{Siembra del material vegetativo}

Habiendo escurrido las estacas se las introdujo a presión lo más rectas posibles, con la misma dirección y teniendo cuidado de no introducir las partes inferiores en las fundas previamente regadas. Igualmente se cubrieron las partes superiores de las estacas con parafina para reducir la evaporación del agua de la estaca y el ingreso de agentes patógenos.

El riego durante los 4 primeros meses de instalado el ensayo se espació ya que se tenía presencia de lluvias en la zona de 3 a 5 veces por semana. En los meses posteriores se hizo de 2 a 4 veces semanales en promedio de acuerdo al requerimiento.

\section{Registro de datos}

A los 90, 120 y 150 días se registró: Porcentaje de prendimiento, número de brotes por estaca y longitud de brotes.

$$
\begin{aligned}
& \text { \% prendimiento }=\frac{\# \text { estacas con brotes } \times 100}{\# \text { total de estacas por tratamiento }} \\
& \# \text { Brotes / estaca }=\frac{\sum \# \text { brotes } / \text { estaca }}{\# \text { estacas con brotes por tratamiento }} \\
& \text { Longitud de brotes }=\frac{\sum \text { longitud }(\mathrm{cm})}{\# \text { estacas prendidas por tratamiento }}
\end{aligned}
$$

\section{Diseño Experimental}

El diseño experimental aplicado es el de bloques completos al azar (BCA) con arreglo combinatorio $6 \times 3$ con 3 repeticiones.

\begin{tabular}{|c|c|c|}
\hline No. & CÓDIGO & DESCRIPCIÓN \\
\hline $\mathrm{T} 1$ & $\mathrm{a} 1 \mathrm{~b} 1$ & $100 \%$ Tierra de páramo + dosis alta A.N.A \\
\hline $\mathrm{T} 2$ & $\mathrm{a} 1 \mathrm{~b} 2$ & $100 \%$ Tierra de páramo + dosis media A.N.A \\
\hline $\mathrm{T} 3$ & $\mathrm{a} 1 \mathrm{~b} 3$ & $100 \%$ Tierra de páramo + dosis baja A.N.A \\
\hline $\mathrm{T} 4$ & $\mathrm{a} 2 \mathrm{~b} 1$ & $100 \%$ Turba + dosis alta A.N.A \\
\hline $\mathrm{T} 5$ & $\mathrm{a} 2 \mathrm{~b} 2$ & $100 \%$ Turba + dosis media A.N.A \\
\hline $\mathrm{T} 6$ & $\mathrm{a} 2 \mathrm{~b} 3$ & $100 \%$ Turba + dosis baja A.N.A \\
\hline $\mathrm{T} 7$ & $\mathrm{a} 3 \mathrm{~b} 1$ & $100 \%$ Paja de páramo + dosis alta A.N.A \\
\hline $\mathrm{T} 8$ & $\mathrm{a} 3 \mathrm{~b} 2$ & 100\% Paja de páramo + dosis media A.N.A \\
\hline $\mathrm{T} 9$ & $\mathrm{a} 3 \mathrm{~b} 3$ & 100\% Paja de páramo + dosis baja A.N.A \\
\hline $\mathrm{T} 10$ & $\mathrm{a} 4 \mathrm{~b} 1$ & $50 \%$ Paja de páramo $+50 \%$ turba + dosis alta A.N.A \\
\hline
\end{tabular}




\begin{tabular}{|c|c|c|}
\hline $\mathrm{T} 11$ & $\mathrm{a} 4 \mathrm{~b} 2$ & $50 \%$ Paja de páramo + 50\% turba + dosis media A.N.A \\
\hline $\mathrm{T} 12$ & $\mathrm{a} 4 \mathrm{~b} 3$ & $50 \%$ Paja de páramo $+50 \%$ turba + dosis baja A.N.A \\
\hline T13 & $\mathrm{a} 5 \mathrm{~b} 1$ & $50 \%$ Paja de páramo $+50 \%$ tierra de páramo + dosis alta A.N.A \\
\hline $\mathrm{T} 14$ & $\mathrm{a} 5 \mathrm{~b} 2$ & $50 \%$ Paja de páramo $+50 \%$ tierra de páramo + dosis media A.N.A \\
\hline $\mathrm{T} 15$ & $\mathrm{a} 5 \mathrm{~b} 3$ & $50 \%$ Paja de páramo $+50 \%$ tierra de páramo + dosis baja A.N.A \\
\hline T16 & a6b1 & $\begin{array}{c}30 \% \text { Paja de páramo }+35 \% \text { tierra de páramo }+35 \% \text { turba }+ \text { dosis } \\
\text { alta A.N.A }\end{array}$ \\
\hline $\mathrm{T} 17$ & $\mathrm{a} 6 \mathrm{~b} 2$ & $\begin{array}{c}30 \% \text { Paja de páramo }+35 \% \text { tierra de páramo }+35 \% \text { turba }+ \text { dosis } \\
\text { media A.N.A }\end{array}$ \\
\hline $\mathrm{T} 18$ & a6b3 & $\begin{array}{c}30 \% \text { Paja de páramo }+35 \% \text { tierra de páramo }+35 \% \text { turba }+ \text { dosis } \\
\text { baja A.N.A }\end{array}$ \\
\hline
\end{tabular}

\section{Esquema del análisis de varianza (ADEVA)}

\begin{tabular}{|c|c|c|c|}
\hline $\mathbf{a}$ & $\mathbf{B}$ & $\mathbf{n}$ & $\mathbf{U E}$ \\
\hline 6 & 3 & 3 & 54 \\
\hline
\end{tabular}

Tabla 3. ADEVA

\begin{tabular}{|lr|c|}
\hline \multicolumn{2}{|c|}{$\begin{array}{c}\text { Fuente de variación } \\
\text { (F.V.) }\end{array}$} & $\begin{array}{r}\text { Grados de libertad } \\
\text { (g.l.) }\end{array}$ \\
\hline Bloques & $($ n-1) & \multicolumn{1}{c|}{$\mathbf{2}$} \\
\hline Tratamientos & $($ ab-1) & 17 \\
\hline Error & $($ ab-1)(n-1) & $\mathbf{3 4}$ \\
\hline TOTAL & $($ abn-1) & $\mathbf{5 3}$ \\
\hline
\end{tabular}

\section{Análisis funcional}

Se utilizó la prueba de separación de medias de Tukey al 5\% para los 18 tratamientos y sus 3 repeticiones.

\section{Resultados y Discusión}

Los datos registrados durante los períodos de evaluación se presentan en gráficos para una mejor comprensión, y para la discusión se han revisado estudios similares y se ha conversado con sus autores para poder interpretarlos acorde a la realidad del presente estudio. 
Gráfico 1. Porcentaje de prendimiento a los 90, 120 y 150 días

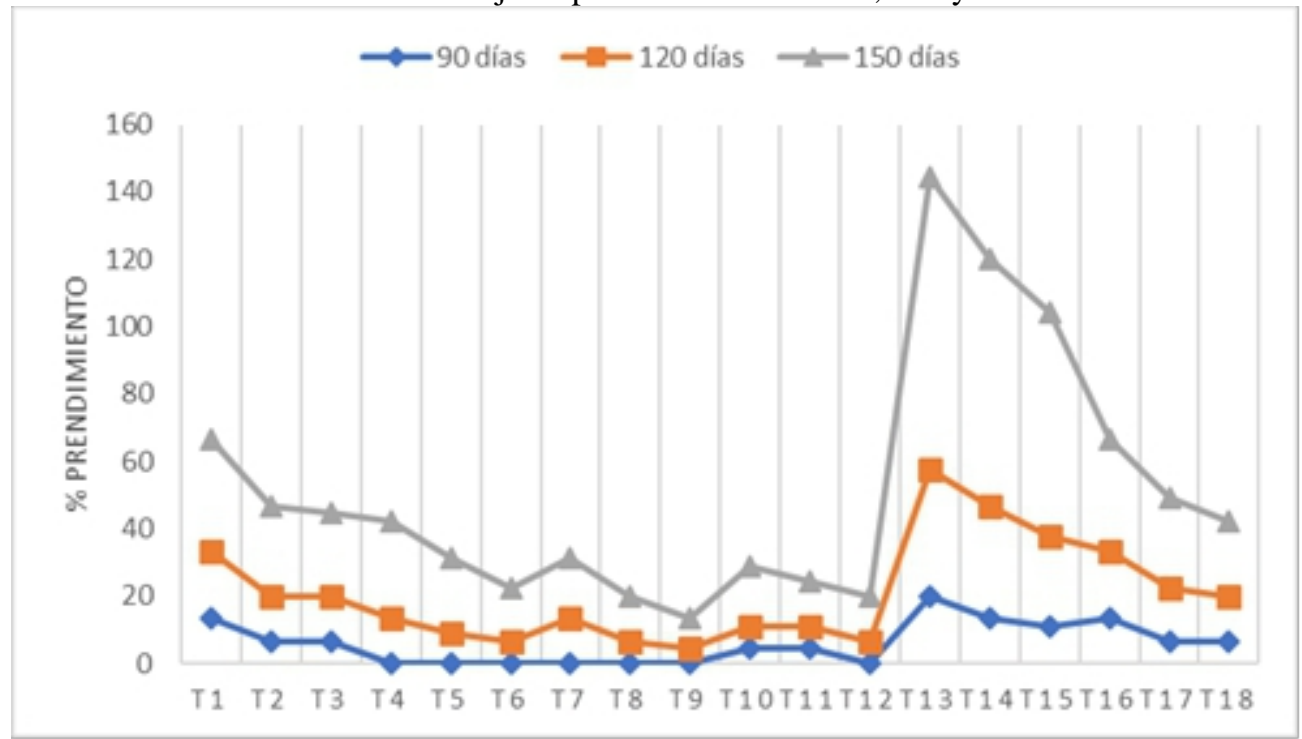

Al realizar la prueba de Tukey al $5 \%$ del porcentaje de prendimiento a los 90 días, se encontró que T13, es el que mayor porcentaje de prendimiento obtuvo con el $20 \%$. Los tratamientos con menor porcentaje de prendimiento fueron T10 y T11 con $4.4 \%$ cada uno; en los tratamientos T4, T5, T6, T7, T8, T9 y T12 se registra un prendimiento nulo. Los sustratos utilizados en los demás tratamientos donde no hubo respuestas favorables respecto al porcentaje de prendimiento a los 90 días estuvieron compuestos de turba y paja de páramo en combinación con las dosis alta, media y baja de auxina.

Aparentemente este comportamiento se debe a que según algunos estudios realizados en otros Vaccinium como V. corymbosum en Chile, existen las micorrizas ericoides presentes en los suelos de crecimiento natural de la especie, suelo que promueve el desarrollo de las estacas cuando no están presentes en sus sitios naturales de crecimiento (Muñoz, 1998).

La tierra de páramo que se usó para la preparación de los sustratos de algunos de los tratamientos fue recogida junto a la zona de crecimiento de Vaccinium floribundum Kunth. La paja de páramo da aireación al sustrato, condición necesaria para el desarrollo de las plantas. Sobre las dosis de $\alpha$ naftalenacético la única experiencia disponible en el país es en el cultivo in vitro, donde TRUJILLO D. 2008, manifiesta tener un mejor respuesta del mortiño a dosis altas de A.N.A.

Los tratamientos 1,16 y 14 , presentaron un $13.3 \%$ de prendimiento; $\mathrm{T} 1$ al no tener un medio de aireación pudo influir en que el prendimiento fuera menor; T16 tiene el sustrato de crecimiento natural del Vaccinium con buena aireación (paja) con una dosis alta de auxina pero la proporción de tierra de páramo es menor y tal vez es la razón por la cual el prendimiento bajó; en el 
siguiente, T 14 tiene dosis media de auxina. Estos tratamientos no difieren significativamente del T15 (11.1\%).

Los tratamientos $2,3,17,18$, que contienen tierra de páramo en menor proporción y menos cantidad de auxina, tienen el menor porcentaje de prendimiento $(6.7 \%)$.

De acuerdo a la prueba de Tukey al $5 \%$ del porcentaje de prendimiento a los 120 días, T13 obtuvo un porcentaje de prendimiento del 37.8\%, lo que no se diferencia significativamente del T14 que obtuvo el $33.3 \%$. Este comportamiento es similar al de los 90 días ya que la especie responde mejor al mismo tipo de sustrato.

Los tratamientos, $11,10,6,12$ y 8 obtuvieron un prendimiento bajo. El porcentaje que alcanzaron fue del $6.7 \%$, lo que no difiere significativamente del T9 con un 4.4\%. En estos tratamientos, los sustratos estuvieron compuestos por paja y turba en combinación con las distintas dosis de auxina. Es importante recalcar que la respuesta de Vaccinium floribundum Kunth. en estos sustratos combinados incluso con la dosis alta de A.N.A es más lenta.

El resto de tratamientos $15,16,1,17,3,2,18,7,4$ y 5 tienen porcentajes que varían entre 26.7, 20.0, 15.6, 13.3 y $8.9 \%$.

El comportamiento de las estacas de mortiño a los 120 días en comparación a los 90 es distinto y no se lo podía predecir, ya que es un arbusto silvestre sin intento de domesticación aún en el país. Después de las evaluaciones es evidente que su desarrollo es lento y que, además de que es necesario que el sustrato contenga tierra del lugar de crecimiento de la especie, la dosis de hormona en estudio influye en su desarrollo. Todas estas variaciones en el comportamiento del porcentaje de prendimiento a los 120 días, se pueden ver en el Gráfico 1.

Habiendo realizado la Prueba de Tukey al 5\% del porcentaje de prendimiento a los 150 días, T13 se mantiene con el mejor prendimiento al igual que en las evaluaciones anteriores con el $86.7 \%$, siguiéndole de cerca los tratamientos 14 y 15 con 73.3 y $66.7 \%$ respectivamente.

Los tratamientos que presentaron los porcentajes de prendimiento más bajos al final de las evaluaciones fueron: T6 con 15.6\%, T11, T12 y T8 con $13.3 \%$ y $\mathrm{T} 9$ con $8.8 \%$.

Al final de los períodos de evaluación del porcentaje de prendimiento vemos que la respuesta de la especie Vaccinium floribundum Kunth fue mejor ante la presencia de la tierra en donde se desarrolla naturalmente la especie, lo que coincide con lo manifestado por MUÑOZ, 1998, y que en aquellos tratamientos donde no tuvo este factor (sustrato de desarrollo natural de la especie), la dosis de ácido $\alpha$ naftalenacético ayudó al prendimiento de las estacas. 
Gráfico 2. Número de brotes por estaca a los 90, 120 y 150 días

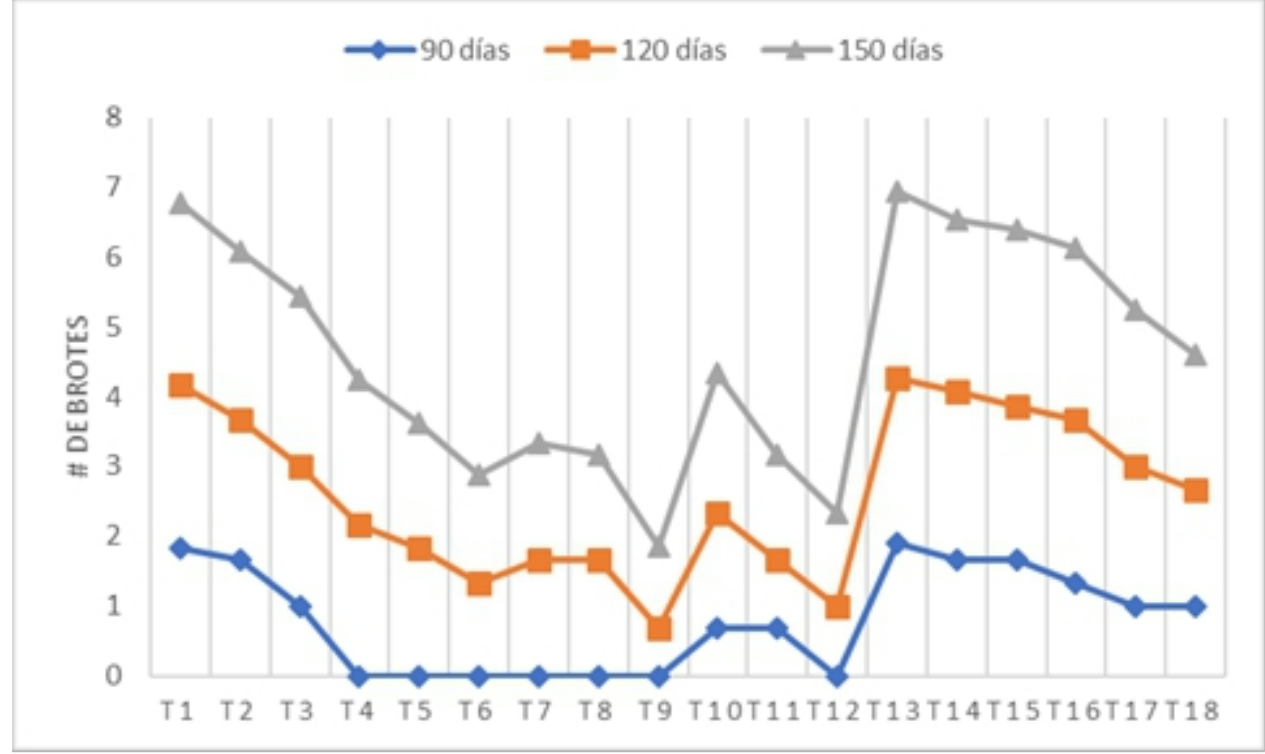

Con la prueba de separación de medias que se aplicó, en este caso la de Tukey al 5\%, T13 es el que en promedio presenta mayor número de brotes a los 90 días, con 1.9 brotes por estaca, sin diferenciarse significativamente del T1 que presentó en promedio 1.8 brotes por estaca. Este caso es similar a los resultados en cuanto al porcentaje de prendimiento ya que en esta variable los tratamientos 13 y 1 son los que ocupan los dos primeros lugares. Estos tratamientos no presentan una diferencia significativa con respecto a los T14, T2 y T15 con promedios de 1.7 brotes por estaca, los mismos que estuvieron compuestos por tierra de páramo y paja de páramo en combinación con las dosis media y baja de $\alpha$ naftalenacético. Los tratamientos restantes $16,3,17$, 18,10 y 11 presentaron en promedio $1.3,1$ y 0.7 brotes por estaca, los mismos que contenían sustratos con paja de páramo, tierra de páramo y turba en combinación con las tres dosis de A.N.A. Los tratamientos 12, 4, 5, 6, 7, 8 y 9, a los 90 días no presentaron brotes; estos contenían turba y paja de páramo en combinación con las tres diferentes dosis de la auxina.

Analizando el comportamiento de la especie a los 90 días en cuanto al número de brotes por estaca, en los tratamientos donde la tierra de crecimiento natural de la especie no formó parte de los sustratos no hubo respuesta a pesar de estar presentes las dosis de auxina, mientras que en los tratamientos donde hubo la presencia de tierra o la combinación con los otros dos sustratos (paja y turba) junto con las dosis de A.NA. la respuesta fue mejor sin superar a la que la especie presentó ante el tratamiento donde el sustrato estuvo compuesto por paja de páramo y tierra de páramo (tierra de crecimiento natural de la 
especie) en proporciones 1:1 en combinación con la dosis alta y media de la auxina.

Al haber realizado la Prueba de Tukey al 5\% del número de brotes por estaca a los 120 días, los que mayor número de brotes presentaron en promedio fueron los tratamientos 14 y 13 , con 2.4 brotes por estaca. Este comportamiento es similar al registrado en el porcentaje de prendimiento a los 120 días.

Los tratamientos T16 y T1 presentaron 2.3 y 2.2 brotes por estaca en promedio.

El número de brotes en promedio de los tratamientos: T2, T17, T3, T5, T18, T7, T8, T10 y T6 fueron de 2, 1.8, 1.7 y 1.3.

Los tratamientos 12, 11 y 9 en cambio fueron los que presentaron los promedios más bajos a los 120 días, que fueron de 1 y 0.66 brotes por estaca.

El comportamiento de la especie en cuanto al número de brotes por estaca a los 120 días fue similar al del porcentaje de prendimiento, ya que la especie reaccionó mejor ante el sustrato conformado por la tierra del lugar de crecimiento natural (tierra de páramo) más paja de páramo en combinación con la dosis alta de A.N.A. Los valores medios se registraron en aquellos tratamientos compuestos por $100 \%$ tierra de páramo o en combinación con paja y turba más dosis alta de la auxina sintética, mientras que los tratamientos donde se presentaron valores bajos estuvieron compuestos por los sustratos: turba y paja con las dosis media y baja de A.N.A. Todo lo descrito permite decir que la presencia de la tierra de crecimiento natural de la especie en combinación con paja de páramo y la dosis alta de la hormona, le da a Vaccinium floribundum Kunth. los medios para un mejor desarrollo, y que la especie reacciona mejor ante la dosis alta de la auxina aunque la tierra de crecimiento natural no esté presente.

Al utilizar la Prueba de Tukey al 5\%, es posible observar que los tratamientos 13 y 1 son los que presentaron mayor número de brotes por estaca en promedio a los 150 días, 2.7 y 2.6 respectivamente.

Los tratamientos T14, T15, T16 obtuvieron en promedio 2.5 brotes por estaca.

Los tratamientos que, sin ser los más bajos, no tuvieron resultados tan favorables son: T2 y T3 con 2.4 , T17 con 2.3 , T4 con 2.1 , T10 con 2 y T18 1.9, T5 con 1.8 , T7 con 1.7 , T6 con 1.6 , T11 con 1.5 y T8 con 1.5 brotes por estaca.

Mientras tanto, los T12 y T9 presentaron los promedios más bajos de número de brotes por estaca, 1.3 y 1.2 respectivamente.

Al inicio de las evaluaciones no hubo presencia de brotes en las estacas que estuvieron plantadas en los sustratos que contenían sólo turba y paja de páramo, o combinadas más las dosis de la auxina. Sin embargo, en las siguientes evaluaciones se puede ver que, aunque los valores de estos 
tratamientos fueron menores en comparación a los demás, la especie tuvo una respuesta. Es decir; el desarrollo del mortiño sin su tierra de crecimiento natural es lento aún si hay la presencia de cualquiera de las tres dosis de auxina. En el tratamiento compuesto de tierra de páramo más paja en proporciones iguales con la interacción de las dosis alta y media de auxina se obtuvieron losmejores resultados.

Gráfico 3. Longitud de Brotes por estaca a los 90, 120 y 150 días

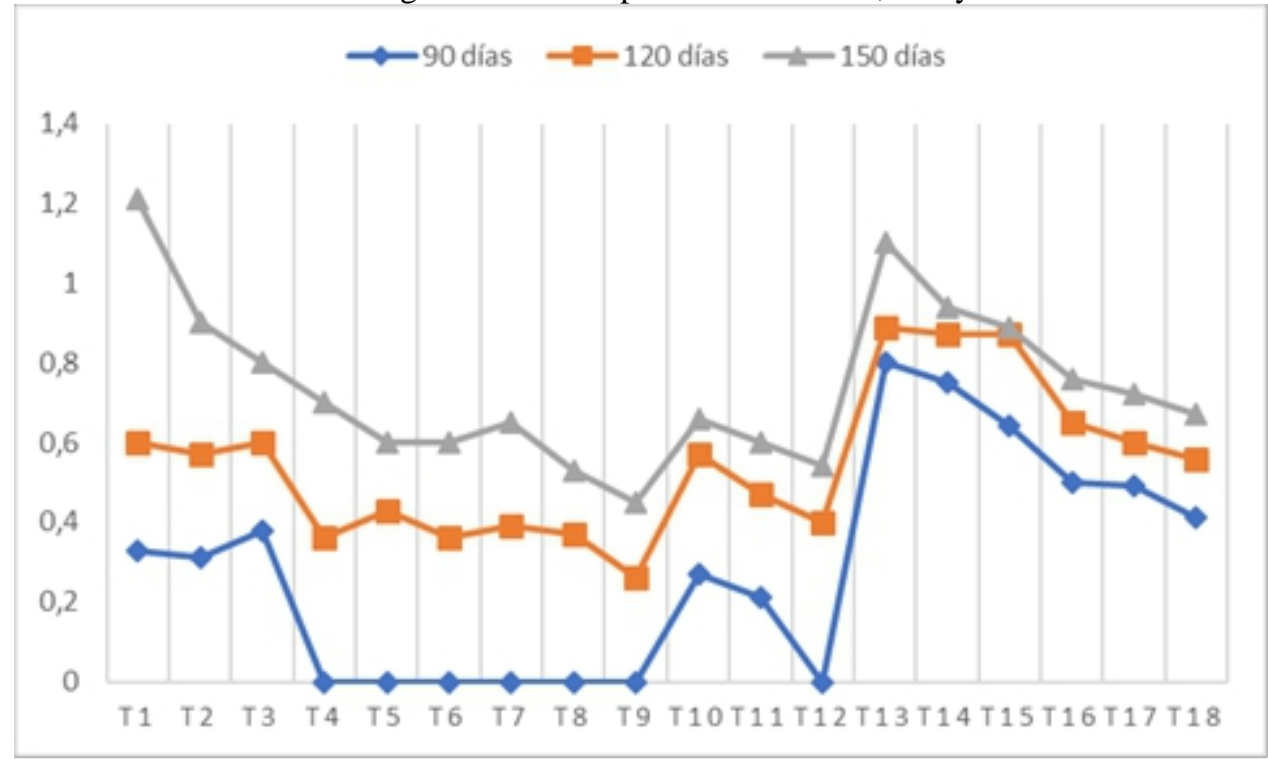

Al haber realizado la Prueba de Tukey al 5\% de la longitud de brotes a los 90 días, los tratamientos que contenían paja de páramo + tierra de páramo en combinación con las dosis media y alta de ácido $\alpha$ Naftalenacético que son los número 13 y 14 son los que mayores longitudes registraron, siendo estas de 0.8 y $0.7 \mathrm{~cm}$ respectivamente.

Los demás tratamientos $15,16,17,18,3,1,2,10$ y 11 presentaron longitudes entre 0.6 y $0.2 \mathrm{~cm}$.

$\mathrm{Al}$ igual que en las otras dos variables a los 90 días, en los tratamientos $12,4,5,6,7,8$ y 9 no hubieron datos que registrar.

Estas variaciones de longitud también podemos observarlas en el Gráfico 3, donde de manera general se puede decir que en aquellos tratamientos donde los sustratos no contenían tierra de crecimiento natural de la especie y que estaban conformados por $100 \%$ turba o paja de páramo, no hubo respuesta, aún con la presencia de las tres dosis de A.N.A. Por el contrario, en el sustrato donde se combinó turba con paja más las dosis alta y media de la auxina hubo una respuesta baja de la especie. En los tratamientos compuestos por tierra de páramo (ó de crecimiento natural) sola o combinada 
con los demás sustratos en las diferentes proporciones indicadas la respuesta del mortiño fue mejor.

A los 120 días, los tratamientos 13, 14 y 15 obtuvieron promedios de longitud de $0.9 \mathrm{~cm}$, siendo los mejores entre los 15 restantes. En los tratamientos T16, T1, T17, T2, T3, T10, T18, T11, T4, T12, T7, T8, T5 у T6 se presentaron longitudes entre 0.7 y $0.4 \mathrm{~cm}$. El tratamiento 9 es el que menor longitud alcanzó registrando un valor de $0.3 \mathrm{~cm}$.

De manera general, observando la respuesta de la especie a los 120 días, se puede decir que el tratamiento en tierra de páramo (ó de crecimiento natural de la especie) con paja en combinación con las tres dosis de A.N.A obtuvieron las longitudes más altas. En aquellos tratamientos compuestos de tierra, sola o combinada con los otros dos sustratos, el comportamiento fue similar entre ellos y los valores que registraron fueron medios, mientras que los sustratos turba y paja de páramo, solos o combinados entre sí, con las diferentes dosis de la auxina, presentaron los valores más bajos en la evaluación.

A los 150 días, los tratamientos que registraron los mejores resultados fueron los números 1 y 13, sin diferenciarse significativamente de los tratamientos 14 . los valores de longitud de brotes que se registraron para $15 \mathrm{y}$ 2fueron 1.2 y $1.1 \mathrm{~cm}$ respectivamente; para los tres siguientes los valores fueron $0.9 \mathrm{~cm}$. Cabe recalcar que esta es la única medición en la que un tratamiento supera al T13.

Los tratamientos que presentaron una longitud de brotes media fueron T3, T16, T17, T4, T18, T10, T7, T5, T11, T6 con valores entre 0.8 y $0.6 \mathrm{~cm}$.

Los tratamientos en los que se registraron los valores más bajos fueron el T12, T8 y T9, los cuales tuvieron brotes cuya longitud oscila entre 0.5 y 0.4. En estos los sustratos estuvieron compuestos de turba y paja de páramo en combinación con la dosis media y baja de la auxina $\alpha$ naftalenacético.

En el caso de esta variable, es la única donde el tratamiento compuesto de $100 \%$ tierra de páramo con dosis alta de la auxina obtuvo el mejor resultado en cuanto a longitud, siguiéndole de cerca los tratamientos que se mantuvieron en los primeros lugares en las demás evaluaciones; es decir, tierra de páramo más paja en proporción 1:1 con las tres dosis de A.N.A. Los valores medios se registraron para los tratamientos compuestos de turba sola o combinada con la tierra y la paja más dosis alta de la auxina, mientras que los valores bajos se presentaron en los tratamientos compuestos de $100 \%$ turba ó paja en combinación con las dosis media y baja de A.N.A.

\section{Conclusiones}

- En base a los resultados obtenidos podemos concluir que el T13 fue mejor en cuanto a porcentaje de prendimiento y número de brotes por estaca en las evaluaciones $(86.67 \%$ de prendimiento y 2.67 
brotes/estaca) y en cuanto a la longitud de brotes el que mejor resultó fue el T1 (100\% tierra de páramo con la dosis alta de la auxina) con un promedio de $1.2 \mathrm{~cm}$. Con esto se comprueba que Vaccinium floribundum Kunth reacciona favorablemente en suelos donde se desarrolla la especie naturalmente y que en combinación con un medio de aireación (paja de páramo) el resultado puede ser mejor.

- Con respecto a las dosis de la auxina sintética ácido $\alpha$ naftalenacético (A.N.A), en todos los casos la especie reaccionó mejor con la dosis alta, e incluso en los sustratos que no contenían tierra de páramo se vio una respuesta favorable aunque un poco baja de $V$. floribundum.

- $\mathrm{Al}$ ser Vaccinium floribundum Kunth un arbusto silvestre en proceso de domesticación aún en el país no se podía predecir su comportamiento, lo que dificultó un poco la investigación. Ahora se evidencia que su desarrollo es lento y que es necesario que el sustrato de propagación contenga tierra del lugar de crecimiento de la especie para mejorar dicho desarrollo.

\section{References:}

1. Acosta, S. (1984). Los páramos andinos del Ecuador. Ed. MAS, Quito- Ecuador. 220 p.

2. Albán, S. \& Burbano, A. (2001). Nuestra vida en los páramos (testimonios). Pp. 123-137 En: MENA, P., G. MEDINA y R.G.M. HOFSTEDE (eds.). Los páramos del Ecuador. Proyecto Páramo y Abya Yala, Quito. $51 \mathrm{p}$.

3. Añazco, M., Loján, L. \& Yaguache, R. (2004). Productos forestales no maderables en el Ecuador (PFNM). Una aproximación a su diversidad y usos. DFC/FAO/MAE/ Gobierno de los Países Bajos, Quito.

4. Boutherin, D. (1994). Multiplicación de Plantas Ornamentales. 225 p. Editorial Acribia. Zaragosa, España.

5. Buzeta, A. (1997). Chile: Berries para el 2000. 133 p. Fundación Chile. Santi Chile.

6. Cleef, A. (1978). Characteristics of Neotropical paramo vegetation and its subantartic relation. Erdwissenschaftliche Forschung.

7. Condiza, C. (1998). Ministerio de Agricultura. Programa Nacional de Transferencia de Tecnología Agropecuaria PRONATTA. Agricultura sostenible.

Colombia. Consultado el 02/08/08. Disponible en http://www.agronet.gov.co/www/docs_si2/20061127145142_Definic ion $\% 20 \mathrm{de} \% 20$ agricultura\%20sostenible.pdf

8. CONVENIO MAG / IICA (2001). Subprograma de Cooperación Técnica (Préstamos BID / MAG 831/OC y 832/OC - EC). 
Identificación de mercados y tecnología para productos agrícolas tradicionales de exportación. Quito.

9. Flores, R. (2005). La situación actual de las negociaciones del TLC entre los Estados Unidos y el Ecuador. Serie Páramo (TLC) 19: 1-22.

10. Freire, F.A. (2004). Botánica sistemática ecuatoriana. Missouri Botanical Garden, pág. 209.

11. Gough, R. (1994). The high bush Blueberry and Its Management. 272 p. Food Product Press, New York, USA.

12. González, L. (2002). PROYECTO PÁRAMO ANDINO - ECOPAR. Propagación y productos del mortiño (Vaccinium floribundum Kunth). Tesis de grado. 100 pp.

13. Hofstede, R.G.M. (1995). Effects of burning and grazing on a Colombian paramo ecosystem. Tesis de doctorado. Universidad de Amsterdam, Amsterdam. 198 p.

14. Luteyn, J.L. (1999). Paramos: a checklist of plant diversity, geographical distribution and geobotanical literature. Memories of the New York Botanical Garden. 84 p.

15. Mena, P \& Balslev, H. (1986). Comparación entre la vegetación de los páramos y el cinturón Afroalpino. AAU Reports. 30p.

16. Mena, P. \& Hofstede, R. (2006). Los páramos ecuatorianos. En: Moraes, M. et al. (Eds.). Botánica Económica de los Andes Centrales. Universidad Mayor de San Andrés. La Paz.

17. Mera, V. (2001). Páramo y prácticas sociales: Caracterización social de los páramos ecuatorianos. Pp. 89-119 En: MENA, P., G. MEDINA \& R.G.M. HOFSTEDE (eds.). Los Páramos del Ecuador. Proyecto Páramo y Abya Yala, Quito.

18. Muñoz, C. (1998). Arándano: Antecedentes generales. Instituto de investigaciones agropecuarias Carrillanca. Seminario: El cultivo del arándano. Temuco, 30 Noviembre, 1 y 2 de Diciembre de 1998. pp. 516. Consultado en Septiembre 2009. Citado en http://ucv.altavoz.net/prontus_unidacad/site/artic/20070723/asocfile/2 00707231002 57/rodrigues_eduardo.pdf

19. Podwojewski, P \& Poulenard, J. (2000). Los suelos de los páramos del Ecuador en Los suelos del páramo. Serie Páramo 5 GTP / Abya - Yala. Quito, Ecuador.

20. Ramsay, P.M. \& Oxley, E.R.B. (2001). An assessment of aboveground net primary productivity in Andean grasslands of Central Ecuador. Mountain Research and Development 21(2): 161-167.

21. Recharte, J. \& Gearheard, J. (2001). Los páramos altamente diversos del Ecuador: Ecología política de una ecorregión. Pp. 55-85 En: MENA, P., G. Medina y Hofstede, R.G.M. (eds.). Los Páramos del Ecuador. Proyecto Páramo y Abya Yala, Quito. 
22. Robles I., Rouillard, S. \& Guaicha, M. (2001). La dinámica de los usos históricos y actuales en el páramo: El ejemplo de Culebrillas, Cañar. Pp. 141-157 En: Mena, P., G. Medina \& R.G.M.

23. Ruiz, P. (2005). Los tratados de libre comercio o la recolonización de América Latina. Serie Páramo (TLC) 19: 47-58.

24. Soto, R. (1993). Efecto de las Características Físicas y Químicas de Diferentes Mezclas de Sustratos en el Crecimiento de Arándano en Maceta. Tesis Ing. Agr. Universidad de Chile, Facultad de Ciencias Agrarias y Forestales. Santiago, Chile.

25. Sudzuki, H.F. (1993). Proyecto: "Frutales menores: Nuevas alternativas de cultivo. 286 p. Universidad de Chile. Convenio: FIAUniversidad de Chile. Santiago, Chile.

26. Vega, E. \& Martínez, D. (1999). Identificación de oportunidades de mercado para servicios ambientales y productos ecológicamente sustentables de los páramos del Ecuador. PROYECTO PÁRAMO. Quito. 48 p.

27. Vega, E. \& Martínez, D. (2000). Productos económicamente sustentables y servicios ambientales del páramo. Serie Páramo (Número monográfico) 4: 1-49. 